\title{
Tarsal buckling como complicação de correção cirúrgica de ptose congênita
}

\author{
Tarsal buckling as a complication of surgery to treat congenital ptosis

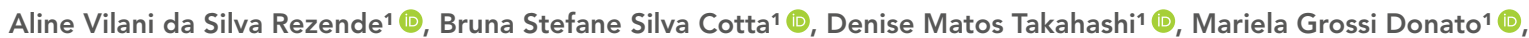 \\ Lívia Maria Neiva Pereira' ${ }^{1}$, Anita Junqueira Leite ${ }^{2}$ (]) \\ ' Instituto de Olhos Ciências Médicas, Belo Horizonte, MG, Brasil. \\ 2 Departamento de Estrabismo Instituto de Olhos Ciências Médicas e Departamento de Plástica Ocular da Ocular Medical Center, Belo Horizonte, MG, Brasil
}

Descritores:

Blefaroptose; Cirurgia plástica; Procedimentos cirúrgicos oftalmológicos; Túnica conjuntiva

Keywords: Blepharoptosis/surgery; Surgery, plastic; Ophthalmologic surgical procedures; Conjunctiva

Recebido: $14 / 08 / 2020$

Aceito:

12/4/2021

Autor correspondente: Mariela Grossi Donato Rua Pouso Alegre, 407 - Floresta CEP: 31110-010 - Belo Horizonte, MG,

E-mail: marielagdonato@gmail.com

Instituição: Instituto de Olhos Ciências Médicas.

Fonte de auxílio à pesquisa: não financiado.

Conflitos de interesse: os autores declaram que não há conflitos de interesses.
O presente trabalho objetivou relatar um caso de tarsal buckling associado a prolapso conjuntival e à inversão de pálpebra superior como complicação da correção cirúrgica de ptose residual. Paciente do sexo feminino, 15 anos, portadora de ptose palpebral residual unilateral em olho direito, secundária à correção parcial da ptose palpebral congênita operada na infância. A segunda abordagem cirúrgica foi realizada com ressecção da aponeurose do músculo levantador da pálpebra superior, que evoluiu com inversão conjuntival da pálpebra superior. A fragilidade estrutural do tarso é a principal hipótese para justificar o tarsal buckling subsequente à cirurgia. Houve resolução completa do tarsal buckling porém houve também persistência da ptose palpebral. O tarsal buckling é, portanto, uma complicação cirúrgica incomum, que pode ocorrer na correção da ptose palpebral, em que há rotação posterior da metade superior do tarso, fazendo com que este se curve verticalmente sobre seu eixo, favorecendo o prolapso conjuntival. A suspeição diagnóstica e a reabordagem cirúrgica precoce favorecem a resolução da condição e previnem possíveis complicações visuais.

\section{ABSTRACT}

This paper aimed to report a case of tarsal buckling associated with conjunctival prolapse and upper eyelid inversion as a complication of surgical correction of residual ptosis. A 15-year-old female patient with unilateral residual eyelid ptosis in the right eye, secondary to partial correction of congenital blepharoptosis operated in childhood. The second surgery was performed with resection of the upper eyelid levator muscle aponeurosis, which progressed to conjunctival inversion of the upper eyelid. The structural fragility of the tarsus is the main hypothesis to justify tarsal buckling after surgery. There was complete resolution of tarsal buckling, but persistence of blepharoptosis. Tarsal buckling is an infrequent surgical complication that can occur in correction of blepharoptosis, when there is posterior rotation of the upper half of the tarsus, causing it to curve vertically on its axis and favoring conjunctival prolapse. Establishing diagnosis and early reoperation favor resolution of the condition and avoid possible visual complications. 


\section{INTRODUÇÃO}

O tratamento de escolha para a correção da ptose palpebral (PP) congênita moderada a grave é a abordagem cirúrgica, dada a maior propensão de causar ambliopia, principalmente quando unilaterais. ${ }^{(1,2)} \mathrm{O}$ procedimento realizado, na maioria das vezes, gera resultados satisfatórios. As complicações são, em geral, transitórias ou discretas, podendo ser encontrados edema, hematomas, hipocorreção, hipercorreção, anormalidade ou assimetria do contorno palpebral. Entretanto, alguns casos podem evoluir com complicações mais graves, como entrópio, prolapso conjuntival, prolapso ou fístula de glândula lacrimal e tarsal buckling, que é o objeto deste estudo, necessitando de reabordagem cirúrgica para correção. ${ }^{(3-5)}$

O Tarsal buckling é a rotação posterior da metade superior do tarso, fazendo com que este se curve verticalmente sobre seu eixo e favorecendo a exposição da conjuntiva. Esse fenômeno pode ser considerado uma complicação que decorre, principalmente, da fixação baixa da aponeurose do músculo levantador da pálpebra superior (MLPS) no tarso durante cirurgia para correção de PP ou fragilidade tarsal. ${ }^{(3,4)} \mathrm{O}$ tarsal buckling propicia o prolapso conjuntival, expondo a conjuntiva do fundo de saco. ${ }^{(2,3)}$ Complicações como exposição e prolapso conjuntivais, bem como o não fechamento ocular adequado, podem culminar em exposição corneana, necessitando de reabordagem cirúrgica precoce, para evitar prejuízos visuais graves. ${ }^{(4,5)}$

Este estudo objetivou apresentar um caso de prolapso conjuntival associado a tarsal buckling de pálpebra superior após reabordagem cirúrgica tardia de PP congênita residual unilateral. Até a presente publicação, este caso era o primeiro de tarsal buckling registrado na literatura brasileira e ocorreu associado com prolapso conjuntiva.

\section{RELATO DO CASO}

Paciente do sexo feminino, 15 anos, com história prévia de ptose congênita no olho direito (OD), operada aos 4 anos de idade com melhora parcial do quadro, procurou o serviço por incômodo estético decorrente da ptose, sem outras queixas. Ao exame, cicatriz visível no sulco palpebral superior direito (PSD), ptose unilateral moderada à direita, euribléfaro de pálpebras inferiores bilateralmente e presença de estrabismo (esotropia de oito dioptrias prismáticas e hipotropia direita de 14 dioptrias prismáticas). Fenda palpebral (FP) de $6 \mathrm{~mm}$ no OD e 9mm no olho esquerdo (OE), função do MLPS de $9 \mathrm{~mm}$ no OD e $16 \mathrm{~mm}$ no OE, distância margem reflexo da pálpebra superior (DMR1) de -1mm em OD e 4mm em OE e reflexo de Bell positivo em ambos os olhos (Figura 1). Acuidade visual com correção (AVCC) 0,6 no OD e 1,0 no OE.

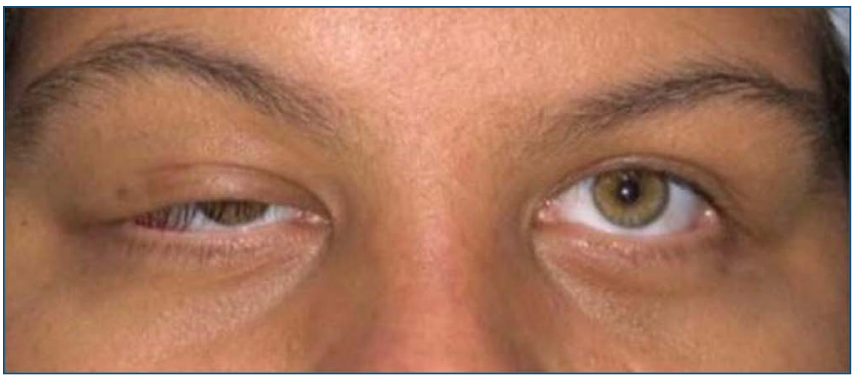

Figura 1. Pré-operatório em posição primária do olhar.

Por apresentar quadro de ptose associada à hipotropia, foi encaminhada para correção do estrabismo, o que foi desaconselhado, com indicação de que a ptose fosse corrigida primeiramente, já que a melhora estética da ptose poderia evitar uma cirurgia de estrabismo, que não era a queixa principal da paciente. Foi realizada, então, a ressecção aponeurótica de 10mm do MLPS de OD.

A paciente evoluiu nos primeiros pós-operatórios (DPO) com discreta exposição da conjuntiva do fundo do saco da margem medial da PSD, com abrasão corneana, edema e equimoses inerentes ao procedimento cirúrgico. A abrasão corneana no local de contato com os cílios invertidos intensificou-se, e a quemose estendeu-se também para a conjuntival temporal. Foi colocada lente de contato terapêutica, com lubrificação intensiva e demais cuidados pós-operatórios habituais.

A paciente evoluiu com melhora gradativa do edema local, FP com boa simetria, discreto lagoftalmo, mas com oclusão palpebral voluntária adequada. Entretanto, a exposição da conjuntiva temporal da PSD persistiu, com aspecto semelhante de conjuntivocálase temporal. No 30o dia após a cirurgia, houve piora importante dessa exposição, com apresentação similar a uma eversão de PSD (Figura 2). Nesse momento, suspeitou-se de tarsal buckling e decidiu-se, então, por nova intervenção cirúrgica.

Durante o ato cirúrgico (Figura 3), à eversão da pálpebra superior, observou-se que a borda superior do tarso da PSD encontrava-se dobrada para baixo, sobre ele mesmo, expondo a conjuntiva do fundo de saco superior e ocasionando o mal posicionamento da pálpebra superior. Foi realizada exploração cirúrgica por via anterior, e o ponto central que ligava a aponeurose do MLPS ao tarso foi encontrado a $8 \mathrm{~mm}$ da margem ciliar. Os quatro pontos de fixação da aponeurose do MLPS no tarso foram removidos, constatando-se a resolução do tarsal buckling ainda no peroperatório. A aponeurose do MLPS foi novamente suturada no tarso em posição anatômica, e a conjuntiva exposta foi suturada com dois pontos de tração no fundo de saco superior. A paciente evoluiu bem no pós-operatório, sem queixas, com reposicionamento da margem palpebral e conjuntival adequados. 
Entretanto, a ptose retornou ao padrão inicial, sendo a FP à direita de $7 \mathrm{~mm}$ no 40o pós-operatório (Figura 4).
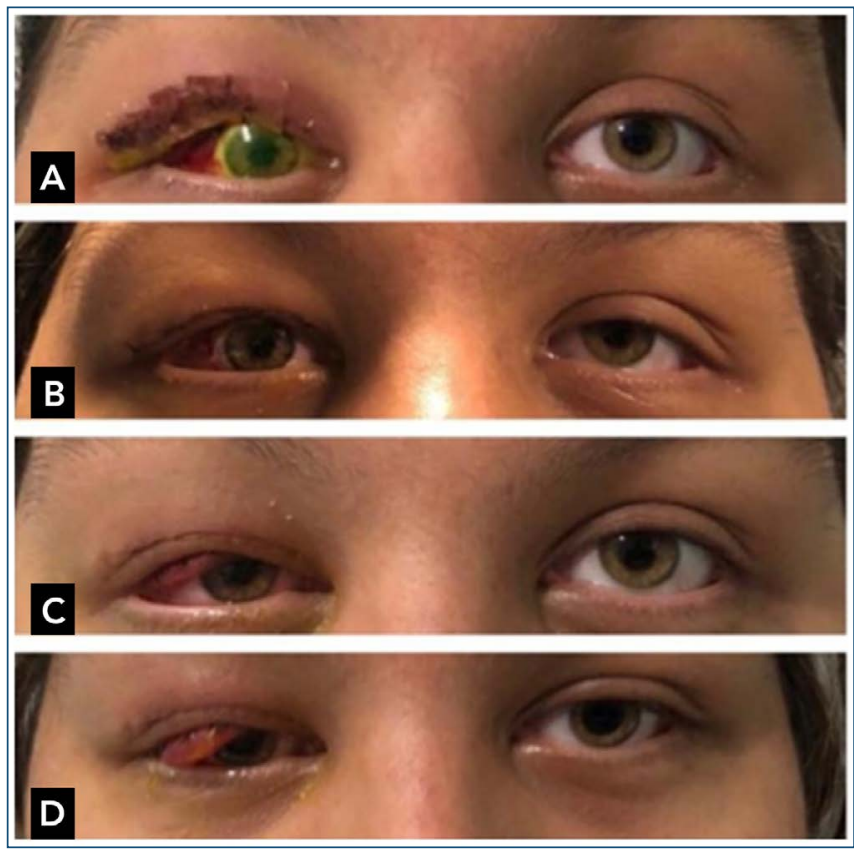

Figura 2. Evolução cirúrgica após a correção de ptose congênita residual associada à hipotropia. (A) Primeiro pós-operatório; (B) sétimo pós-operatório mostrando discreta exposição da conjuntiva do fundo do saco; (C) $15^{\circ}$ pós-operatório com exposição de conjuntiva evidente; (D) $30^{\circ}$ pós-operatório com piora da exposição conjuntival.
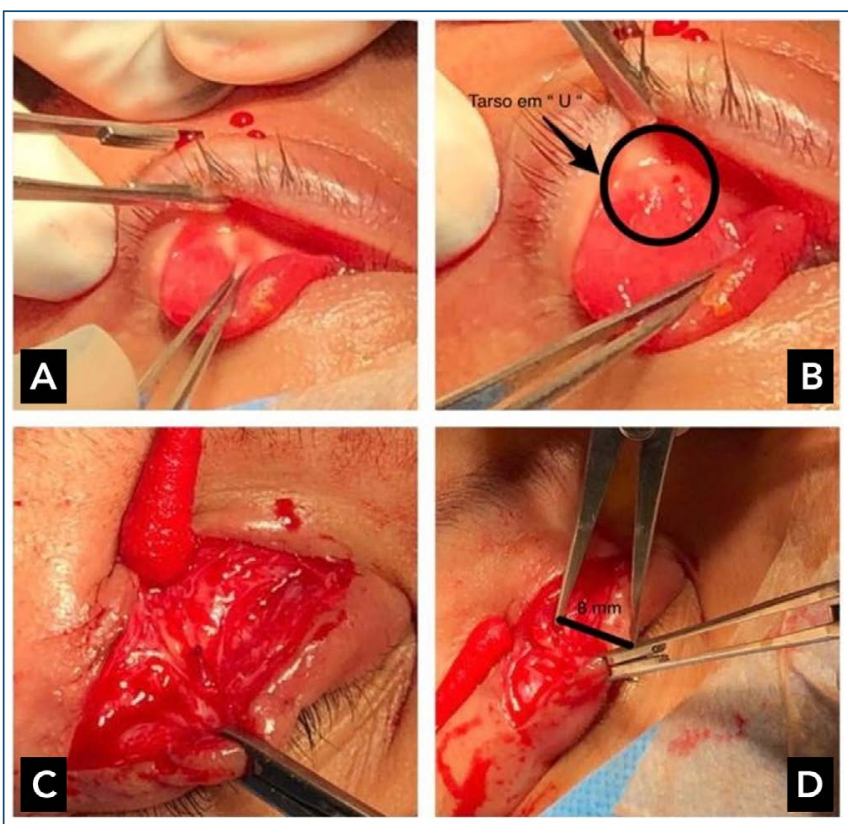

Figura 3. Fotodocumentação mostrando (A) a porção interna do tarso; (B) tarso com deformidade em "u" (seta) na área central; (C) vista anterior da pálpebra superior mostrando a abertura da pele na região da prega palpebral superior e localização da sutura entre o músculo levantador da pálpebra superior e o tarso; (D) demonstração da medida da posição do músculo levantador da pálpebra superior a $8 \mathrm{~mm}$ em relação à margem ciliar.

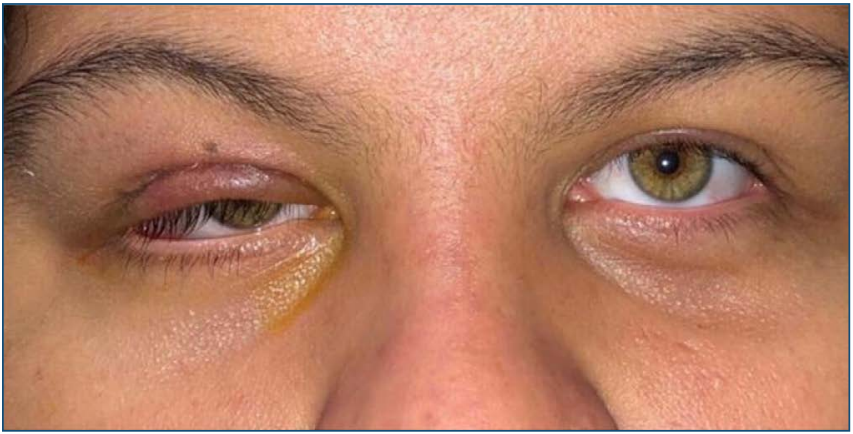

Figura 4. Aspecto pós-cirúrgico decorridos 40 dias da primeira cirurgia. A paciente continuou com a ptose palpebral e recuperou a posição da margem palpebral, com bom posicionamento da conjuntiva.

Estudo submetido ao Comitê de Ética em Pesquisa - Ciências Médica, inscrito no número CAAE 37204920.4.00005134.

\section{DISCUSSÃO}

Os autores apresentam um caso raro de tarsal buckling que ocorreu em uma adolescente reoperada de PP congênita em OD. O quadro surgiu após a segunda cirurgia para correção de ptose residual, tendo sido a primeira correção de ptose congênita realizada na infância, e necessitou de uma terceira intervenção cirúrgica para resolução do tarsal buckling.

A PP congênita consiste na diminuição vertical da FP por descida da pálpebra superior. Independente da técnica cirúrgica empregada e da execução adequada para correção, há possibilidade de complicações, como hipercorreção, ectrópio, pregas assimétricas na pele e ptose residual, sendo essa última o desfecho observado após a primeira intervenção cirúrgica deste caso. ${ }^{(6)} \mathrm{A}$ paciente em questão apresentava hipotropia associada à PP, quadro que pode se relacionar a pior resultado da correção cirúrgica. A possível dupla paralisia de elevadores (reto superior e levantador da pálpebra) é uma condição congênita que limita o resultado da cirurgia de ptose. ${ }^{(7)}$

Diante do desconforto estético importante gerado em paciente adolescente e diante da boa função do MLPS (9mm OD), nossa primeira opção foi referenciar a paciente a um estrabólogo que opinou pela correção da pálpebra e não indicou a correção da hipotropia. Optou-se, então, por realizar a ressecção da aponeurose do MLPS como nova tentativa de melhora do quadro, o que gerou as outras repercussões pós-operatórias. ${ }^{(2,3)}$

O prolapso conjuntival como complicação pós-operatória pode ocorrer após diversas abordagens cirúrgicas, como ressecção do músculo levantador da pálpebra superior, orbitotomia, cirurgias de retina e correção de fratura de assoalho orbitário, especialmente em casos em que a dissecção 
anatômica é extensa e associada a fatores inflamatórios inerentes ao procedimento, os quais favorecem a transudação e o acúmulo de líquido no espaço subconjuntival, levando ao edema e à projeção da conjuntiva dos fundos de saco. ${ }^{(2,5)}$

O tratamento dessa complicação, visando evitar a progressão, bem como queratinização da conjuntiva exposta, pode ser clínico, com reposicionamento da conjuntiva, ou envolver procedimentos como injeção subconjuntival de lidocaína e epinefrina, blefarorrafias ou suturas para fixação conjuntival, a depender da intensidade do caso. ${ }^{(2,4)}$

A paciente apresentou, além do precoce prolapso conjuntival temporal superior à direita, piora progressiva, associada ao tarsal buckling de PSD. Embora raro, o tarsal buckling pode ocorrer como desfecho indesejado da correção de ptose, sendo importantes o reconhecimento e o tratamento dessa enfermidade, visando minimizar o acometimento corneano. . $^{(3,4,8)}$

Em geral, há evidências dessa ocorrência quando a aponeurose do MLPS é inserida muito inferiormente na face anterior do tarso superior - abaixo do terço inferior -, gerando uma força de rotação sobre ele mesmo, ${ }^{(4)} \mathrm{com}$ possibilidade de se instalar o entrópio, ou o ectrópio palpebral. Apesar de ser a causa mais comumente observada, a fixação baixa da aponeurose do MLPS não ocorreu nesse caso, fato constatado na reabordagem cirúrgica, quando se detectou a localização adequada das suturas tarsais, dispostas a $8 \mathrm{~mm}$ da margem ciliar.

Outra característica pouco abordada na literatura que pode desestabilizar a posição da pálpebra é a fragilidade tarsal, traduzida pela espessura menor do tarso (especialmente se menor que 50\%). O adelgaçamento tarsal pode gerar resultantes vetoriais que agiriam como agonistas do músculo orbicular pré-tarsal, causando inversão do tarso e, consequentemente, um entrópio. ${ }^{(3)}$ Esse tarso mais fino e menos resistente poderia apresentar rotação posterior de sua metade superior, fazendo com que o tarso se curve verticalmente sobre seu eixo, em um movimento denominado tarsal buckling. ${ }^{(3)}$ Assim, a debilidade estrutural do tarso poderia ser o fator mais relevante para a ocorrência do tarsal buckling nessa paciente.

Por fim, a dobra tarsal favorece o prolapso conjuntival, expondo a conjuntiva do fundo de saco..$^{(2,3,9)}$ A conjuntiva exposta é o suficiente para que o mecanismo de exsudação de fluidos ocorra, com prolapso da conjuntiva do fundo de saco superior. Instala-se um ciclo vicioso, no qual o tarsal buckling leva ao prolapso de conjuntiva, o qual por sua vez, acentua o tarsal buckling.

Pouco se discute, na literatura, sobre a fragilidade tarsal como fator causal para ocorrência de tarsal buckling. (3,6) Essa alteração tarsal é referida principalmente em pacientes idosos, portadores de ptose involucional..$^{(9)}$ No caso aqui apresentado, questiona-se a ocorrência de doença do colágeno associada e/ou debilidade tarsal secundária à intervenção cirúrgica prévia.

Os autores apresentaram uma paciente com ptose palpebral associada à hipotropia, na qual observaram um fenômeno raro conhecido como tarsal buckling após reabordagem cirúrgica da ptose que ocorreu associada com o prolapso de conjuntiva. O fator determinante para o tarsal buckling foi apontado como a fragilidade tarsal. Outros estudos e relatos precisam ser discutidos para que se elaborem opções de tratamento cirúrgico e condução clínica em casos semelhantes.

\section{REFERÊNCIAS}

1. Lima AL, Dantas MC, Alves MR. Doenças externas oculares e córnea. 4a ed. Rio de Janeiro: Cultura Médica; 2016. Vol. 1. [Série Oftalmologia Brasileira].

2. Pujari A, Shashni A, Bajaj MS, Samdani A. Tarsal buckle with conjunctival prolapse following levator plication for unilateral congenital ptosis. BMJ Case Rep. 2018;2018:bcr-2017-223743.

3. Shafi FK, Mehta P, Ahluwalia HS. Upper lid entropion post ptosis correction: is tarsal buckling the cause? Orbit. 2012;31(4):246-8.

4. Patipa M, Wilkins RB. Vertical tarsal buckling as a complication of levator aponeurosis repair for acquired blepharoptosis. Am J Ophthalmol. 1984;97(1):93-9

5. Sabino LR, Montenegro AA, Guimarães BD, Farias AB, Silva JJ. Entrópio palpebral severo pós cirurgia de correção de ptose. Rev Bras Oftalmol. 2019;78(2):141-3.

6. Cardoso AM, Cardoso J, Marques N, Miranda A, Pereira M, Fonseca T, et al. Ptose congénita: da clínica ao tratamento. Rev Soc Port Oftalmol. 2014;38(1):49-52

7. Carvalho KM, Zin A, Bicas HE, Dias CR. Oftalmologia pediátrica e estrabismo. 4a ed. São Paulo: Conselho Brasileiro de Oftalmologia; 2017. Vol. 1. [Série Oftalmologia Brasileira].

8. Dollin M, Oestreicher JH. Adult-onset exposure keratitis after childhood ptosis repair with frontalis sling procedure. Can J Ophthalmol. 200944(4):412-6.

9. Malhotra R, Salam A, Then SY, Grieve AP. Visible iris sign as a predictor of problems during and following anterior approach ptosis surgery. Eye (Lond). 2011;25(2):185-91. 